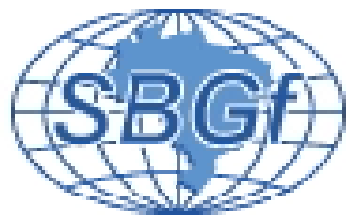

\title{
Detecção de eventos sísmicos ao longo do tempo através da transformada wavelet associada à mapas auto-organizáveis de Kohonen
}

Marcílio C. Matos, Instituto Militar de Engenharia e Pontifícia Universidade Católica do Rio de Janeiro (PUC-Rio), Paulo L. M. Osório, PUC-Rio, e Roberto Fainstein, NExT/Schlumberger

Copyright 2006, SBGf-Sociedade Brasileira de Geofísica

Este texto foi preparado para a apresentação no Il Simpósio de Geofísica da Sociedade Brasileira de Geofísica, Natal, 21-23 de setembro de 2006. Seu conteúdo foi revisado pela Comissão Tecno-científica do II SR-SBGf mas não necessariamente representa a opinião da SBGf ou de seus associados. E proibida a reprodução total ou parcial deste material para propósitos comerciais sem prévia autorização da SBGf

\section{Resumo}

A new alternative to detect time-lapse seismic effects is presented herein. We propose to use clustering of self organizing maps (SOM) associated with the wavelet transform to detect time-lapse changes. The wavelet transform is used to detect seismic traces singularities of each time-lapse 3D cube which are then classified using the clustering of the SOM. In this fashion, seismic trace singularities that have changed along time are identified. The technique was then successfully applied to the Troll West gas province, offshore Norway.

\section{Introdução}

Existem diversas formas de comparação entre dados sísmicos 3-D adquiridos ao longo do tempo. As avaliações qualitativas e quantitativas de projetos de sísmica ao longo do tempo são implementados com o objetivo de detectar mudanças que possam ser evidenciadas em dados sísmicos 3-D que estejam diretamente associadas com a produção do reservatório. Normalmente, a principal ferramenta utilizada é o sinal sísmico chamado de $4 \mathrm{D}$ que é o obtido através da diferença cross-equalizada entre diferentes dados sísmicos 3-D. Entretanto, a cada dia novas tecnologias são disponibilizadas no mercado quebrando antigos paradigmas. Novas informações

Neste contexto, este artigo propõe o uso de técnicas de reconhecimento de padrões para identificar efeitos de lapso sísmico no tempo. Especificamente, atributos sísmicos obtidos para cada cubo sísmico 3-D são comparados ao longo do tempo utilizando técnicas estatísticas de análise multi-atributos ou de redes neurais. Desta forma, espera-se obter indicadores de anomalias 4-D geradas em função das mudanças das propriedades físicas do reservatório devido à produção de óleo/gás.

Neste trabalho, a técnica de agrupamento dos mapas auto organizáveis de Kohonen, SOM (Vesanto e
Alhoniemi, 2000) foi utilizada para detecção e classificação das fácies sísmicas, enquanto, a tranformada wavelet foi utilizada para extração dos atributos sísmicos que variam ao longo do tempo. A transformada wavelet (WT) foi usada para detector as singularidades dos traços sísmicos de cada cubo sísmico 3-D. Matematicamente, sabe-se que os traços sísmicos podem ser caracterizados por suas singularidades (Hoekstra, 1996) e elas são menos sensíveis a variações na interpretação sísmica. Após a detecção das singularidades de cada traço sísmicos, elas são classificadas utilizando o algoritmo de agrupamento do SOM. Desta forma, os traços sísmicos que sofreram variação ao longo do tempo são identificados.

Além da eficácia do método quando aplicado a análise de fácies sísmica 3-D (Matos et al., 2003), esta metodologia de classificação também auxilia na identificação do número de fácies.

Portanto, este artigo apresenta uma metodologia de classificação Taplicado à análise sísmica 4-D.

\section{Análise de fácies sísmica utilizando mapas auto organizáveis de Kohone (SOM) associado a singularidades de traços sísmicos detectados através da transformada wavelet}

Uma forma de se estimar o número de fácies sísmicas e de classificar os dados sísmicos de forma não supervisionada é através do agrupamento dos mapas auto organizáveis de Kohonen (Matos et al., 2003). A Figura 1 sintetiza o processo, para quando os atributos sísmicos de entrada são as formas de onda, i.e., as amplitudes sísmicas instantâneas, para um sinal sísmico sintetizado a partir de um modelo com três diferentes fácies. Entretanto, quando os atributos são extraídos a partir de uma interpretação ruidosa, os resultados da análise de fácies são ruins, conforme pode ser ilustrado na Figura 2. Uma forma de se evitar este problema é através da utilização das singularidades dos traços sísmicos detectados através dos módulos dos máximos da transformada wavelet (WTMMLA) como atributos sísmicos (Matos et al., 2003). Este processo é detalhado em Matos et al. (2006) e está esquematizado na Figura 3: 

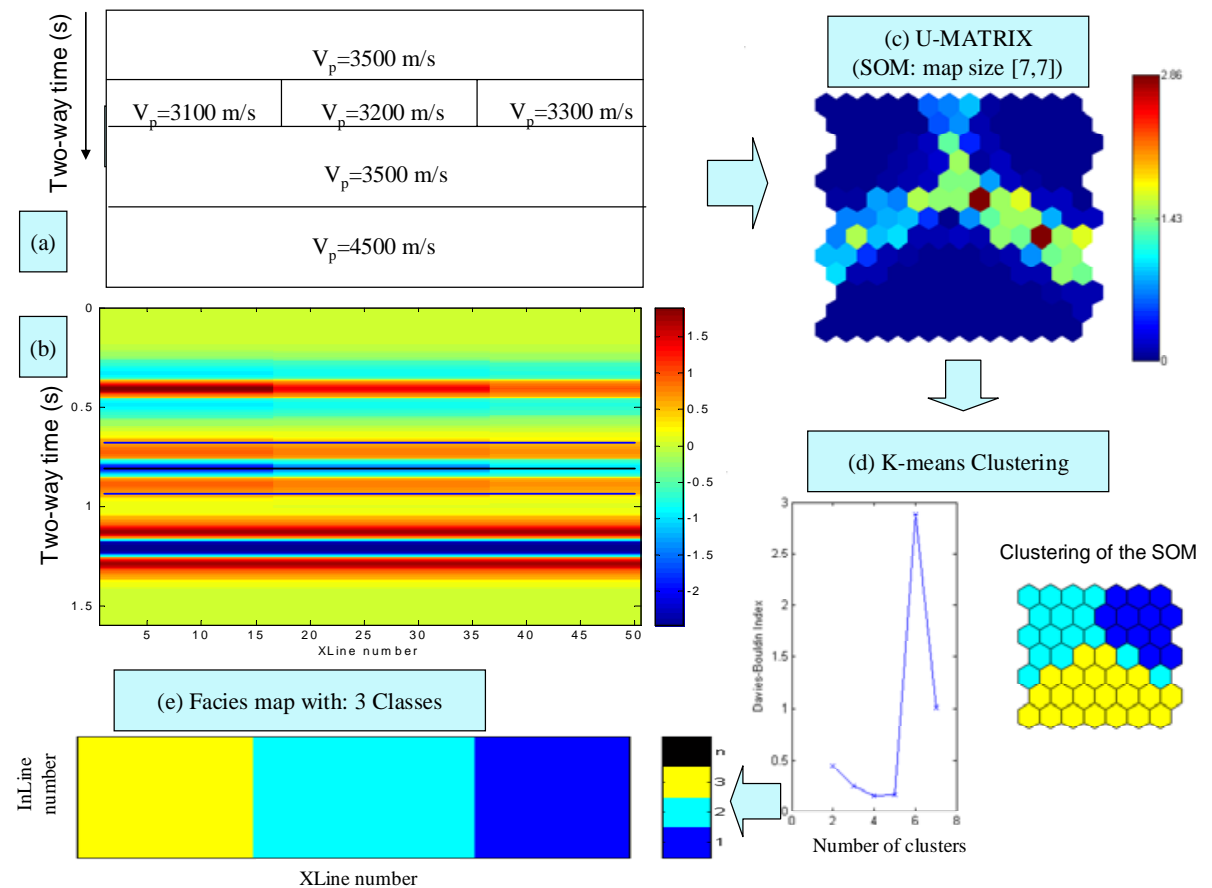

Figure 1: (a) Modelo de velocidade; (b) Sinal sísmico sintetizado e a segmentação temporal, o color bar representa a amplitude; (b) Umatrix, o color bar representa a distancia; (c) Davies Bouldin Index (DBI); (d) Mapa de fácies sísmicas, o color bar representa as classes.

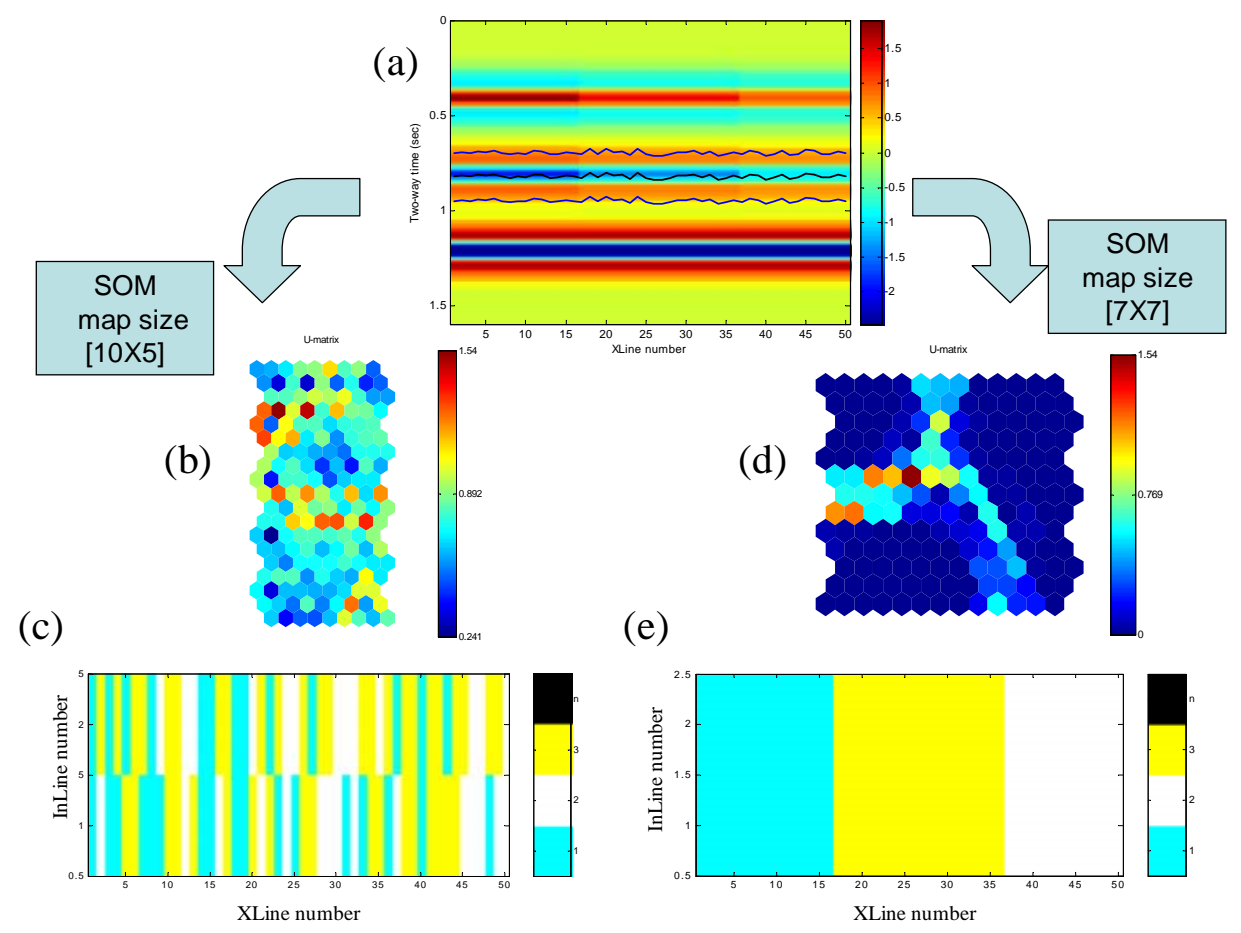

Figure 2: Análise de fácies sísmicas dos dados sintetizados, segmentados a partir de uma interpretação ruidosa; (a) sinal sísmico, o color bar representa a amplitude; (b) U-matrix, o color bar representa a distancia; (c) mapa de fácies sísmicas utilizando a forma de onda como atributo de entrada, o color bar representa as classes; (d) U-matrix, o color bar representa a distancia; (e) mapa de fácies sísmicas utilizando os atributos obtidos a partir da WTMMLA, o color bar representa as classes. 


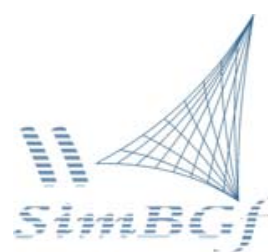

$\|$ Simpósio Brosilero de Geófísico ,21 a 23 de Setembro de 2006, Natal - RN

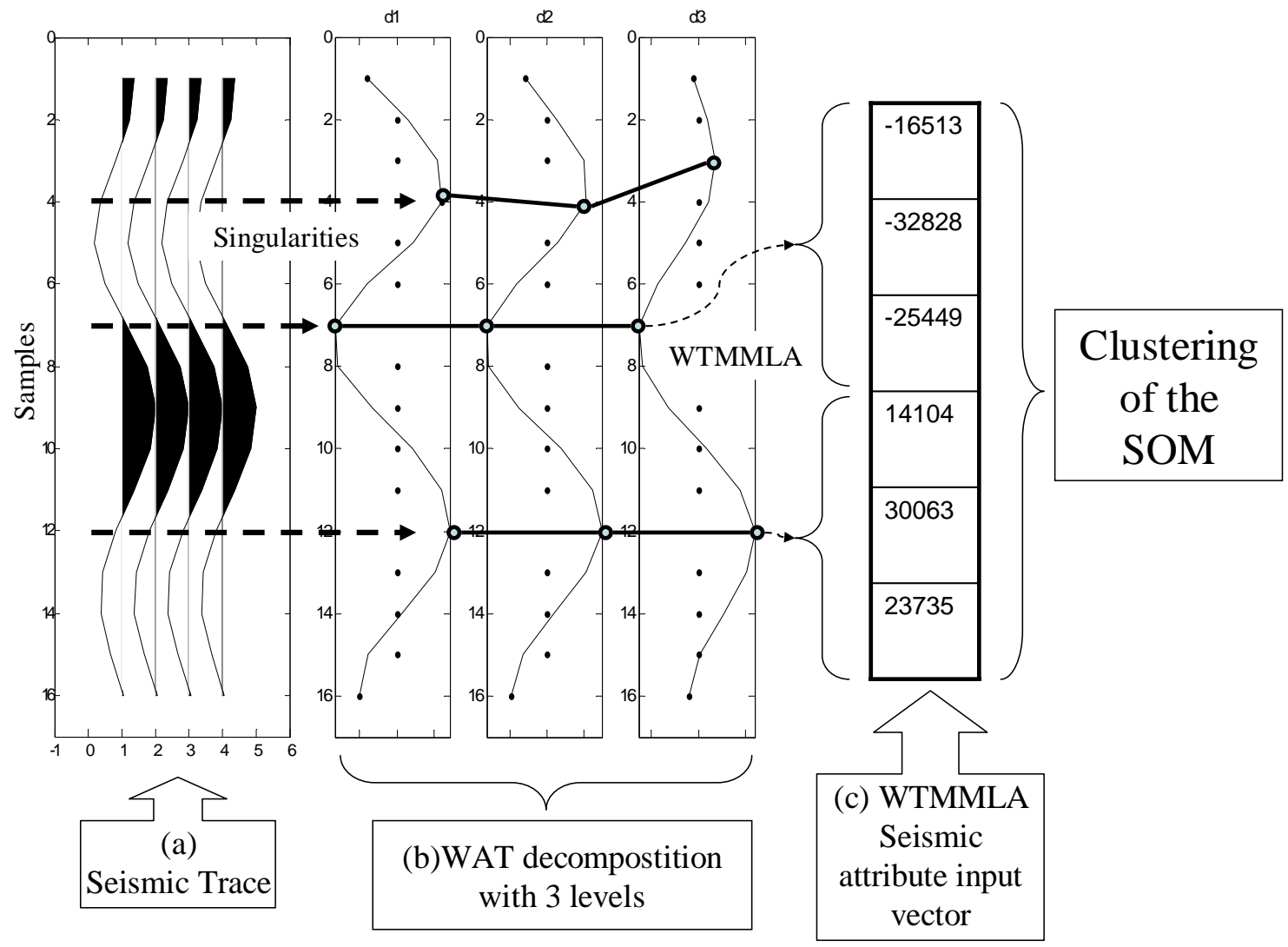

Figure 3: (a) Traço sísmico com 16 amostras no tempo extraídas em torno do flatspot; o traço sísmico foi repetido quatro vezes; (b) Decomposição WAT em três níveis e WTMMLA associada às singularidades dos traços sísmicos; (c) O vetor de atributos WTMMLA mostra as duas WTMMLA mais significantes, mantendo a localização no tempo. Este vetor é usado como entrada para o algoritmo de agrupamento do SOM.

\section{Detectando efeitos sísmicos ao longo do tempo utilizando o SOM e WTMMLA}

As técnicas de reconhecimento de padrões sísmicos são normalmente utilizadas para identificação de efeitos sísmicos ao longo do tempo, através da procura por atributos sísmicos obtidos na região do reservatório a efeitos de produção (Sonneland et al., 1997), (Lucet e Fournier, 2001) e (Oldenziel et al., 2002).
Matematicamente, sabe-se que os traços sísmicos podem ser caracterizados por suas singularidades e podem ser detectados e caracterizados pelos módulos dos máximos da transformada wavelet (WTMMLA) (Matos et al., 2003). De fato, neste trabalho propomos o uso de todo o WTMMLA extraído de cada traço sísmico delimitado por uma janela em torno do horizonte de interesse. Estes atributos foram usados como entrada para o sistema de classificação de padrões baseado no agrupamento do SOM. Após o treinamento, cada 
cubo sísmico 3-D é classificado utilizando o SOM obtido a partir do treinamento com os cubos sísmicos originais concatenados. A metodologia proposta é detalhada na Figura 4.

Como as singularidades dos traços sísmicos são menos sensíveis a interpretações ruidosas, a vantagem do método é a habilidade de identificar quais singularidades estão associadas a mudanças devido a produção.

\section{Aplicando a metodologia proposta ao campo gigante de gás Troll oeste}

O método proposto foi aplicado ao campo de gigante de gás Troll Oeste, localizado na costa da Noruega, onde o principal objetivo da análise sísmica ao longo do tempo é o de estudar e identificar possíveis movimentações no contato óleo-água (Bertrand and Bannister, 2005). Nesta análise, 16 amostras das amplitudes sísmicas foram retiradas em torno do flatspot e então as duas principais curvas associadas às singularidades foram detectadas utilizando três níveis da transformada wavelet. A Figura 5 ilustra os resultados obtidos. A identificação do número de fácies existente entre os dois cubos 3-D foi realizada através da análise do agrupamento do SOM. Comparando os resultados mostrados nas Figuras $5 b$ e 5c, com os atributos sísmicos ilustrados nas Figuras $6 a$ e $6 b$, obtidos do sinal de diferença 4D, pode-se verificar que as mesmas regiões identificadas com 0 sinal $4 \mathrm{D}$ são também identificadas com a técnica proposta e estão indicadas com círculos tracejados nas Figuras $5 b$ e 5c. Além destas regiões, pode-se também observar nas regiões marcadas com círculos brancos outros efeitos de lapso no tempo não observados diretamente no sinal sísmico 4D.

\section{Conclusões}

Observações da representação em todo o domínio tempo-freqüência podem ser interpretadas como um padrão sísmico e utilizada com entrada para um sistema de classificação de padrões sísmicos utilizando o agrupamento do SOM. Desta forma, é possível se estimar, de forma semi-automática, o número de efeitos 4D e classificá-los.

Os resultados obtidos confirmam que a metodologia proposta pode ser uma alternativa eficaz para a detecção de efeitos de lapsos sísmicos no tempo.

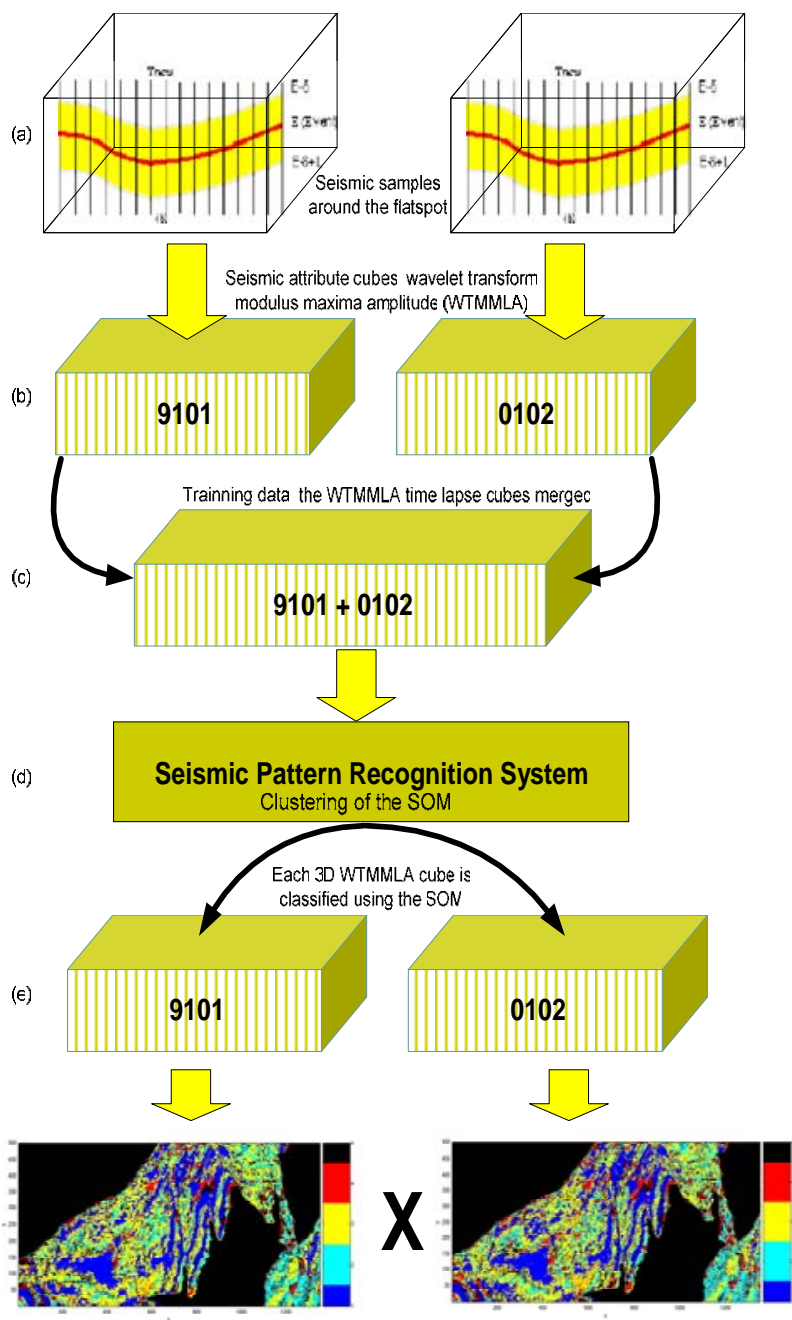

Comparing the different Seismic facies maps with time, the time lapse effects could be identified.

Figure 4: (a) Amostras sísmicas em torno do flatspot; (b) cubos WTMMLA; (c) Cubo utilizado para treinar a SOM; (d) O agrupamento do SOM; (e) Processo de classificação. 
(a)

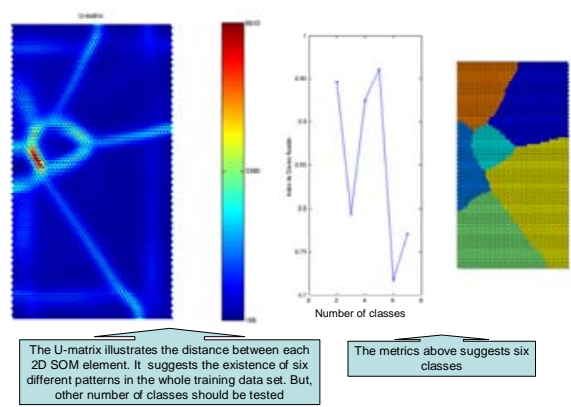

(b)
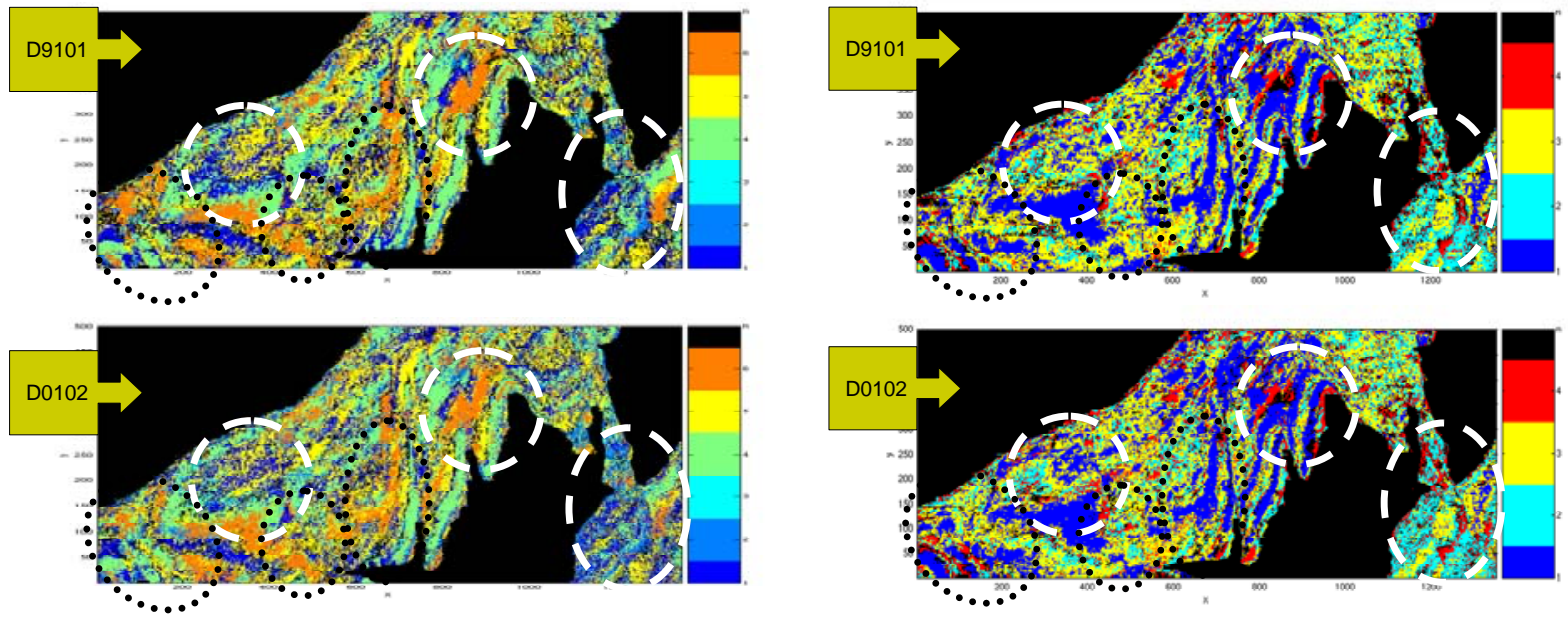

Figure 5: (a) Agrupamento do SOM utilizando as duas WTMMLA mais importantes de cada traço sísmico como atributos de entrada. A U-matrix ilustra a distância entre cada elemento do SOM 2-D. Ela sugere a existência de seis padrões diferentes em todo o conjunto de dados. Entretanto, outro número de classes pode ser testado; (b) Mapa de fácies sísmicas utilizando 6 classes; (c) Mapa de fácies sísmicas utilizando 4 classes.

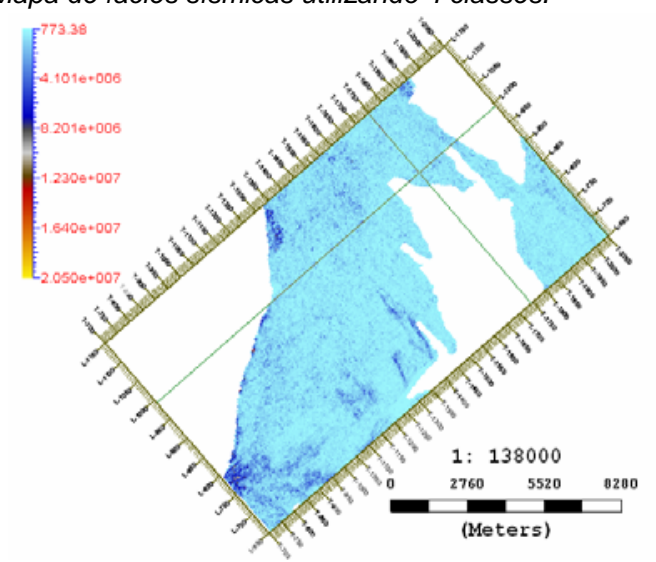

(a)

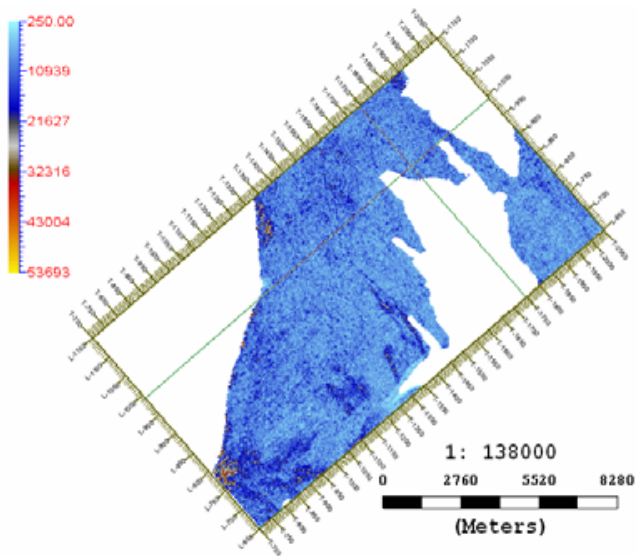

(b)

Figure 6: Atributos sísmicos 4D: a) Energia em torno do flatspot; b) Valor absoluto integrado em torno do flatspot. 


\section{Agradecimentos}

Os autores agradecem à Norsk Hydro e a seus parceiros no campo de Troll na Noruega pela permissão de uso dos dados sísmicos e pela divulgação dos resultados apresentados neste trabalho. O primeiro autor também agradece à Nork Hydro pelo suporte financeiro e pela oportunidade de desenvolver este trabalho durante estágio de pesquisa em outubro de 2002.

\section{Referências}

Bertrand, A. and D. Bannister [2005] Enhanced 4D processing and quantitative analysis on Troll west using multiple vintages of legacy data: First Break, 23.

Hoekstra, E.V. [1996] Multiscale Analysis of Seismic Data by the Wavelet Transform: Msc. Thesis, Delft University of Technology.

Lucet, N. and F. Fournier [2001] 4D data interpretation through seismic facies analysis: 71th Annual Meeting SEG, Expanded Abstracts.

Matos, M.C., P.L.M. Osorio, and P.R.S. Johann [2003] Using wavelet transform and self-organizing maps for seismic reservoir characterization of a deep-water field, Campos basin, offshore Brazil: 65th Annual Meeting,

EAGE.

Matos, M.C., P.L.M. Osorio, and P.R.S. Johann [2006] Using wavelet transform and self-organizing maps for seismic reservoir characterization: Submitted to Geophysics. (Available at: http://www.lps.ele.pucrio.br/-marcilio).

Oldenziel, T., P. Medahl, H. Ligtenberg, P. Digranes and L.K. Stronen [2002] Multiattribute analysis of 4D anomalies using pattern recognition technology: 72th Annual Meeting SEG, Expanded Abstracts.

Sonneland, L., H.H. Veire, B. Raymond, L. Pedersen, S. Ryan and C. Sayers [1997] Seismic reservoir monitoring on Gulfaks: The Leading Edge.

Vesanto, J., Alhoniemi, E. [2000] Clustering of the SelfOrganizing Map: IEEE Transactions on Neural Networks, Volume 11, Number 3, pp. 586-600. 